\title{
Hacia la transformación de los estudiantes: un proceso transdisciplinario para la educación superior
}

\author{
Towards the transformation of students: a transdisciplinary process for \\ higher education
}

\section{Rumo à transformação dos alunos: um processo transdisciplinar para o ensino superior}

\author{
Graciela Salgado-Escobar \\ Instituto Politécnico Nacional, UPIICSA, Departamento de Estudios \\ Profesionales Genéricos, México \\ gsalgadoe@ipn.mx \\ https://orcid.org/0000-0003-2144-4965 \\ Mario Aguilar-Fernández \\ Instituto Politécnico Nacional, UPIICSA, Sección de Estudios de \\ Posgrado e Investigación, México \\ maguilarfer@ipn.mx \\ https://orcid.org/0000-0003-2621-8692
}

\section{Resumen}

Configurar un mundo sostenible requiere nuevas formas de pensar y actuar donde la educación para el desarrollo sostenible sea una propuesta para el cambio. Esto, sin embargo, trae consigo retos para la educación superior, ya que implica formar estudiantes con competencias transdisciplinares. Por eso, el objetivo de esta investigación fue diseñar un proceso transdisciplinario para la educación superior centrado en el estudiante y en el vínculo IES, industria y sociedad, con base en la teoría holónica, la metodología de los sistemas suaves y la metodología de stage-gates con el fin de desarrollar el pensamiento sistémico, el trabajo colaborativo y la creatividad en los futuros profesionales y responsables de la construcción del tejido social en un mundo sostenible. Para ello, se diseñó una investigación 


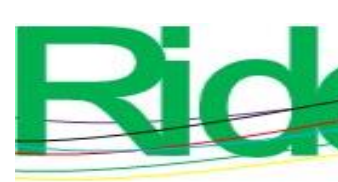

Revista Iberoamericana para la
Investigación y el Desarrollo Educativo
ISSN $2007-7467$

cualitativa, de tipo documental, la cual se llevó a cabo en dos fases: en la primera se efectuó una revisión de la frontera del conocimiento en las publicaciones relativas a la transdisciplina y la educación superior en la Web of Science de los últimos cuatro años, mientras que en la segunda se desarrolló el proceso, el cual se configuró en siete etapas, cimentado en el sustento teórico del constructivismo y el sustento pedagógico del enfoque por competencias. Se obtuvo como resultado el proceso transdisciplinario para la educación superior (PTD-ES), configurado por cuatro momentos que abarcan desde lo simple hasta lo complejo: I) disciplinario, II) multidisciplinario, III) interdisciplinario, IV) transdisciplinario; y tres compuertas para valorar el nivel de competencia que desarrollaron los estudiantes en cada momento.

Palabras clave: competencias transdisciplinarias, desarrollo sostenible, disciplinariedad, enseñanza superior.

\section{Abstract}

Shaping a sustainable world requires new ways of thinking and acting, so education for sustainable development is a proposal for change, yet it brings with it challenges for higher education, since it involves training students with transdisciplinary skills. So it is objective of this research, to design a transdisciplinary process for higher education, focused on the student and the higher education, industry and society link, based on holistic theory, soft systems methodology and stage-gates methodology, to develop systemic thinking, collaborative work and creativity in future professionals and those responsible for building a sustainable world. It is a qualitative research, documentary type, which was carried out in two phases: the first, was a review at the frontier of knowledge, in publications related to transdiscipline and higher education in the Web of Science of the last four years; and the second, was the design of the process, which was shaped in seven stages, based on the theoretical support of constructivism and the pedagogical support of the approach by competencies The result was the Transdisciplinary Process for Higher Education (PTD-ES), configured for four moments, ranging from the simple to the complex: I) disciplinary, II) multidisciplinary, III) interdisciplinary, IV) transdisciplinary; and three gates to assess the level of skills that students developed at each point.

Keywords: transdisciplinary skills, sustainable development, disciplinary, higher education. 


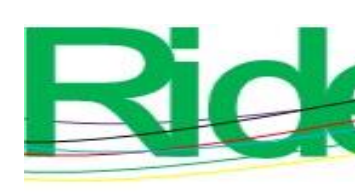

Revista Iberoamericana para la Investigación y el Desarrollo Educativo ISSN $2007-7467$

\section{Resumo}

A construção de um mundo sustentável requer novas formas de pensar e agir, onde a educação para o desenvolvimento sustentável é uma proposta de mudança. Isso, no entanto, traz consigo desafios para o ensino superior, pois implica formar alunos com competências transdisciplinares. Portanto, o objetivo desta pesquisa foi delinear um processo transdisciplinar para o ensino superior centrado no aluno e na articulação entre a IES, a indústria e a sociedade, com base na teoria holônica, na metodologia dos soft systems e na metodologia dos stage-gates ordenados. desenvolver o pensamento sistêmico, o trabalho colaborativo e a criatividade dos futuros profissionais e responsáveis pela construção do tecido social em um mundo sustentável. Para tanto, foi elaborada uma pesquisa qualitativa, do tipo documental, realizada em duas fases: na primeira, uma revisão da fronteira do conhecimento em publicações relacionadas à transdisciplina e ao ensino superior na Web of Science das quatro últimas anos, enquanto na segunda foi desenvolvido o processo, que se configurou em sete etapas, com base no suporte teórico do construtivismo e no suporte pedagógico da abordagem por competências. O resultado foi o processo transdisciplinar para o ensino superior (PTD-ES), configurado por quatro momentos que vão do simples ao complexo: I) disciplinar, II) multidisciplinar, III) interdisciplinar, IV) transdisciplinar; e três portas para avaliar o nível de competência que os alunos desenvolveram a cada momento.

Palavras-chave: competências transdisciplinares, desenvolvimento sustentável, disciplinaridade, ensino superior.

Fecha Recepción: Marzo 2021

Fecha Aceptación: Octubre 2021

\section{Introducción}

Es innegable que el mundo en el que hoy vivimos está inmerso en diversas problemáticas que están situando a la sociedad en riesgo de desaparecer. Cuestiones como la pobreza, el desempleo, la migración, el abandono escolar, el déficit comercial, el narcotráfico internacional, el calentamiento global o la aparición de nuevas enfermedades (actualmente, el SARS-CoV-2) ya no pueden concebirse como algo aislado, sino como parte de un sistema dinámico y complejo, del cual formamos parte, por lo que nos concierne a "todos" aprender a vivir conjuntamente de modo sostenible (Organización de las Naciones Unidas para la Educación, la Ciencia y la Cultura [Unesco], 2020). Sin embargo, lo que vemos depende de la manera en que estemos preparados para verlo (Kant, 2003). 

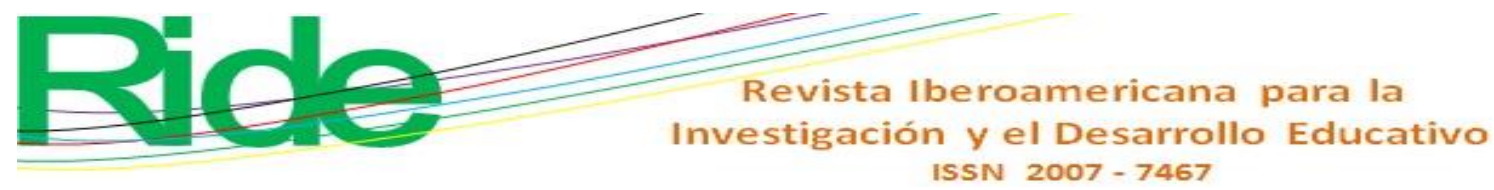

Sin duda, la educación es la fuerza vital para el desarrollo, pues a través de ella se logra conservar y transmitir su particularidad tanto espiritual como física de una sociedad (Jaeger, 2001). Una propuesta para transitar a un mundo sostenible es la educación para el desarrollo sostenible (EDS), que promueve una formación transformadora e integral, la cual engloba pedagogía, contenidos, resultados y entornos de aprendizaje (Unesco, 2020), en donde las instituciones de educación superior (IES) juegan un papel transcendental para impulsarla (Rammel, Velázquez y Mader, 2015) por considerárseles fuentes principales en la generación de conocimiento para dar respuesta a los cambios actuales provocados por la progresiva complejidad de la sociedad (Jantsch, 1972; Klein, 2004).

Empero, el generar más conocimiento disciplinar, innovación y contar con más expertos educados que transfieran conocimientos no es suficiente para transitar a la sostenibilidad, ya que se requiere incorporar el diálogo entre los diferentes ámbitos del conocimiento, los valores, la participación y aprendizaje social (Unesco, 2020). La estructura e interdependencia de los problemas de la sociedad rebasan los prismas disciplinares, lo que conduce al cuestionamiento de cómo formar a los estudiantes en esta transformación económica y social, sin precedentes (Klein, 2004; Morin, 1996).

Esto representa un desafío para la educación superior (ES), ya que para comprender este "mundo complejizado" es imprescindible concebir la racionalidad humana desde otra visión, lo que exige cambios en la manera de educar que conduzcan a otra manera de pensar, comprender y valorar "más allá de" (Nicolescu, 1999). Ante esta realidad, la transdisciplina (TD) aparece como una solución (Lang et al., 2012) a los problemas actuales de la sociedad (Jantsch, 1972; Nicolescu, 1999).

Si bien la TD en la ciencia de la sostenibilidad es cada vez más relevante y efectiva en donde se interrelacionan los desarrollos técnicos, económicos y sociales, con los valores y culturas (Klein, 2004), en la ES aún se concibe de manera limitada (Fiala et al., 2018). Por ello, es necesario que el proceso de enseñanza y aprendizaje se reconfigure con un enfoque transdisciplinario (Acevedo-Osorio, Hofmann-Souki y Cruz Morales, 2020), el cual debe estar vinculado a la EDS, y ambos ser una parte conceptual y sistémica de la cultura educativa de la ES (Biberhofer y Rammel, 2017).

No obstante, la TD no es fácil de lograr (Lang et al., 2012). Aunque "la disciplinariedad, la pluridisciplinariedad, la interdisciplinariedad y la transdisciplinariedad son cuatro flechas de un solo y mismo arco: el del conocimiento" (Nicolescu, 1999, p. 37), 


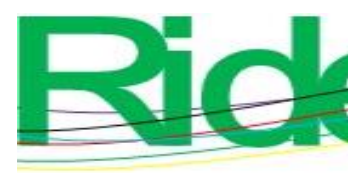

Revista Iberoamericana para la
Investigación y el Desarrollo Educativo
ISSN $2007-7467$

la TD se enfrenta a una diversidad de obstáculos, principalmente, al "pensamiento único" de la disciplinariedad, la cual no es adversaria, sino complementaria (Nicolescu, 1999).

Es manifiesto que la investigación TD difiere del trabajo disciplinario, por lo que se deben desarrollar competencias específicas (Lang et al., 2012; Fiala et al., 2018), lo que conduce a implementar formas de educación que desarrollen en los estudiantes y docentes los términos interdisciplina y $T D$, así como la capacidad de juicio en situaciones complejas y dinámicas (Klein, 2004), que difícilmente en los formatos de aprendizaje tradicional se pueden desarrollar (Acevedo-Osorio et al., 2020).

\section{La TD y la EDS: competencias que promueven en los estudiantes}

El concepto transdisciplina emerge en el primer Seminario Internacional sobre Interdisiciplinariedad, que se llevó a cabo en 1979 copatrocinado por la Organización de Cooperación y Desarrollo Económico (OCDE), con reflexiones como la de Jean Piaget y Erich Jantsh (Klein, 2004) acerca de la manera en que la visión disciplinaria fragmenta el conocimiento, lo que imposibilita el entendimiento de las realidades del mundo complejo (Piaget, 1972) y de la necesidad de desarrollar un sistema para la educación TD, centrado en la combinación sistémica de múltiples niveles de investigación, educación e innovación (Jantsch, 1972).

La TD, por ende, se configura bajo la inquietud de aquello que inicia en las disciplinas, las interrelaciona y las traspasa para comprender el mundo actual (Nicolescu, 1999), por lo que son considerados niveles de la TD: la disciplina, multidisciplina y la interdisciplina. En el nivel de disciplinariedad, se estudia un tema de investigación desde un solo campo de estudio y nivel de la realidad, a través de un pensamiento simple. En el multidisciplinar, varias disciplinas persiguen un objetivo en común, bajo su independencia metodológica, epistemológica y conceptual. En el de interdisciplinariedad, se trasfieren metodologías de una disciplina a otra que pueden tener grado de aplicación, epistemológico o generación de nuevas disciplinas. Y en el TD es globalmente abierto, pues exige unidad del conocimiento y una nueva perspectiva para coexistir (Nicolescu, 1999).

Dado que la EDS hace énfasis en la necesidad de desarrollar una manera diferente de enseñar y aprender entre diversas disciplinas, y más allá de ellas, de tal forma que se desarrolle una nueva visión para construir un mundo sostenible (Unesco, 2020), esta refleja en sí misma un enfoque TD (Biberhofer y Rammel, 2017). Así, la TD es concebida como 


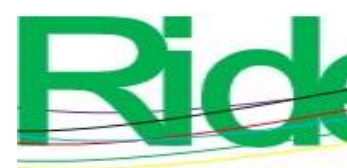

\section{Revista Iberoamericana para la Investigación y el Desarrollo Educativo ISSN $2007-7467$}

una postura transformadora de la educación, que procura que los estudiantes aprendan a ser, aprendan a conocer, aprendan a hacer, aprendan a vivir juntos y, principalmente, aprendan a transformarse a sí mismos y a la sociedad (Unesco, 2020). Para aplicarlo, se requiere un ambiente dinámico que propicie el entorno de aprendizaje, fundamentalmente configurado en el aprendizaje basado en problemas, la interconexión entre y más allá de las disciplinas y la integración de partes interesadas (Biberhofer y Rammel, 2017).

La enseñanza transformadora busca animar a los estudiantes a explorar el cambio epistémico, por lo que no puede limitarse solo a la ES, sino abarcar todo el entorno TD entre la ciencia y la sociedad, de tal manera que se comparta e intercambie conocimiento científico y no científico entre los interesados para enfrentar los desafíos de la sostenibilidad. Por eso, la enseñanza no puede limitarse a la adquisición de conocimientos e intervención cognitiva, ya que es indispensable que incluya el intercambio y perfeccionamiento de aptitudes prácticas junto con la traducción de valores (Sipos, Battisti y Grimm, 2008).

Visto que la educación es capaz de transformar y crear nuevas formas de vivir (Morin, 1999), se espera que los procesos de enseñanza y aprendizaje en la ES desarrollen individuos capaces de abordar problemas sociales multifacéticos (Mokhele y Pinfold, 2020) para enfrentar los desafíos de sostenibilidad (Unesco, 2020). Desde el enfoque constructivista, estos procesos deben suscitarse en ambientes de aprendizaje auténticos y vinculados con problemas (Piaget, 1972).

Por otro lado, el enfoque pedagógico por competencias plantea que las instituciones educativas configuren planes de estudios interdisciplinarios, flexibles, amplios y centrados en contenidos que promuevan el razonamiento, abiertos a las contribuciones de quienes aprenden y que logren integrar el aprendizaje formal y el informal. En otras palabras, que permitan desarrollar las competencias primordiales para la vida y el trabajo, esto es, la creatividad, la capacidad de emprendimiento, la reflexión, la comunicación, el pensamiento crítico, la resolución de problemas, la colaboración, la asunción de riesgos, la innovación y el metaconocimiento (Scott, 2015).

Así, se considera que los estudiantes deben desarrollar, a través de la investigación TD, cuatro capacidades (Muhar, Visser y van Breda, 2013): la capacidad de integración, que implica el valorar las diversas contribuciones disciplinarias y no disciplinarias; la capacidad de comunicación, que supone el generar y negociar nuevos conceptos y representaciones para la comprensión colectiva; la capacidad metodológica innovadora, que exige la incorporación de metodologías, y la capacidad de aprendizaje recíproco en diversos contextos y ámbitos 


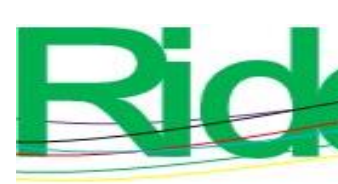

Revista Iberoamericana para la
Investigación y el Desarrollo Educativo
ISSN $2007-7467$

múltiples perspectivas, a llevar procesos de análisis, debate y a tomar decisiones para mejorarla. Por eso, se concibe como un ciclo de aprendizaje interminable (Checkland, 2000).

El SSM está definido por siete principios: i) una situación problemática percibida, ii) percibida de manera distinta por personas con diferentes visiones del mundo, iii) contendrá a la gente tratando de actuar a propósito, iv) por lo tanto, hacer modelos de actividad con propósito como lo perciben las diferentes visiones del mundo, v) utilizar el modelo como fuente de preguntas para plantear la situación problemática, estructurando así una discusión, vi) encontrar versiones de la situación por cambiar con las diferentes visiones del mundo podría vivir con vii) implementar cambios para mejorar (estar listo para iniciar el proceso de nuevo). Estos siete principios subyacen claramente en las cuatro acciones que definen la forma del SSM: 1) averiguar sobre una situación problemática; 2) hacer que los modelos sean relevantes para explorarla basados en diferentes visiones del mundo; 3) cuestionar la situación usando los modelos con el fin de encontrar un cambio deseable y posible, y 4) definir/tomar medidas para cambiar la situación para mejor. El séptimo principio define por sí mismo una quinta acción que garantiza el ciclo alrededor de los cuatro primeros, es decir, la reflexión crítica sobre todo el proceso. Esta quinta acción está en un nivel diferente de las otras cuatro. Es la actividad que asegura que las lecciones aprendidas sean capturadas (Checkland, 2000).

Por otra parte, la propuesta de la metodología stage-gate - aun cuando está enfocada en mejorar la eficacia y eficiencia de la gestión del proceso de nuevos productos- bien puede aplicarse a la TD. Es una metodología que consiste en una serie de etapas (stage), donde el equipo del proyecto realiza el trabajo, obtiene la información necesaria y realiza la integración y análisis de datos para llevar el proyecto al siguiente punto de partida o de decisión, seguido de compuertas (gate), donde se toman decisiones para seguir a la siguiente etapa o repetir, para lo cual se debe definir lo que se tiene que entregar, los criterios para evaluar el proyecto y el resultado final junto con un plan de acción para la siguiente etapa (Cooper, 2008). 


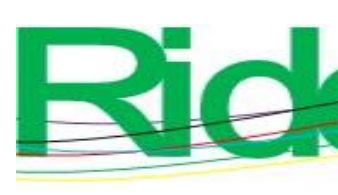

Revista Iberoamericana para la
Investigación y el Desarrollo Educativo
ISSN $2007-7467$

\section{Investigaciones previas}

A partir de la revisión de literatura realizada en la WoS, se percibe en las publicaciones, de manera general, una visión compartida por parte de los autores en la necesidad de promover en la ES un proceso de enseñanza y aprendizaje TD que incite a los estudiantes al descubrimiento y la reflexión crítica para transitar hacia la sostenibilidad. Dichas publicaciones se clasificaron, de acuerdo con su propuesta, en cuatro categorías: la primera se compone por aquellas que proponen un diseño de un programa o curso de estudios, como son propuestas que integran técnicas modernas de gestión y conceptos de sostenibilidad con la dinámica de la industria y la sociedad (Century, Ferris y Zuo, 2020; Contreras, Jiménez, Browne y Oliva-Figueroa, 2020; Mkhize, 2019; Mokhele y Pinfold, 2020; OlivaFigueroa et al., 2018; Roysen y Cruz, 2020; Rupnik y Avsec, 2020; Serviss y Voss, 2019).

Asimismo, propuestas centradas en el aprendizaje basado en proyectos (Marcos, De Castro y Martín-Peña, 2020; Tasdemir y Gazo, 2020), centradas en desarrollar en los estudiantes las competencias indispensables para realizar la investigación TD (Acevedo-Osorio et al., 2020; Echegoyen-Sanz y Martin Ezpeleta, 2019), y en las que se integra la práctica con la política, la ciencia y la tecnología (Fiala et al., 2018).

La segunda categoría integra propuestas enfocadas en institucionalizar la TD en la ES, entre estas, una investigación que analiza el proceso de institucionalización de la TD en las universidades desde la perspectiva neoinstitucionalista (Baptista y Rojas-Castro, 2020). Asimismo, una propuesta en la que se reflexiona sobre las implicaciones de la inter y la TD como un enfoque metodológico donde se discute, como cuestión de fondo, las crisis actuales del conocimiento disciplinario (Khoo et al., 2018). Otras acerca de la eficacia de la aplicación del enfoque transdisciplinar (Barakhsanova et al., 2020; Garutsa y Mahlangu, 2019), y sobre la efectividad de los módulos TD cuando se imparten en línea con teorías constructivistas moderadas (basadas principalmente en Jean Piaget y John Dewey), en línea con teorías transdisciplinarias y mediante una combinación de ambas teorías (Keller et al., 2019), así como sobre las variaciones en las que los profesores de diferentes materias experimentan la enseñanza colaborativa en el contexto de todo un proyecto de desarrollo educativo escolar (Norden, 2018).

La tercer categoría corresponde a las propuestas sobre la TD y la vinculación de la ES. En esta se hallan trabajos que examinan los desafíos relacionados con la sostenibilidad en Austria y se analizan las oportunidades para que la ES atienda estos desafíos mediante la 


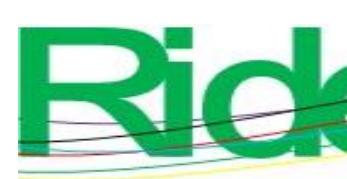

Revista Iberoamericana para la Investigación y el Desarrollo Educativo ISSN $2007-7467$

colaboración transdisciplinaria entre la ciencia y la sociedad, es decir, las partes interesadas en el cambio sostenible (Biberhofer y Rammel, 2017), en sesiones de capacitación innovadoras para educadores universitarios (Meyer et al., 2017). Además, se identifica una propuesta de un modelo de coordinación de la investigación transdisciplinaria utilizando la iniciativa de salud pública TD en Estados Unidos (Holhl, Knerr y Thompson, 2019).

Por otro lado, existe un trabajo que analiza y describe cómo los centros de investigación TD - Real-world Laboratories (RwLs) - favorecen los procesos de aprendizaje y transformación a través de un espacio híbrido que facilita el intercambio de conocimiento entre la esfera académica y otras de la sociedad (Beecroft, 2018).

La cuarta categoría engloba propuestas sobre la TD y la integración del conocimiento, entre estas se encuentra la que visualiza espacios prácticos educativos ambientales TD, conceptuales y aplicados, en el contexto de knowledge-based society/economy (KBS/E) (Pop, Fotea y Fotea, 2018). Además, una propuesta que conceptualiza las constelaciones de prácticas TD creadas sobre una perspectiva de práctica social, en la que el conocimiento y el aprendizaje se realizan en prácticas interrelacionadas que pueden abarcar múltiples campos como el profesional, el educativo y el de investigación (Laasch et al., 2020).

Asimismo, propuestas acerca de la influencia de la confianza en el intercambio de conocimiento entre los miembros de una comunidad de práctica transdisciplinar (tecnológico-educativa) (Hernández-Soto, Rodríguez-Medina y Gutiérrez-Ortega, 2020). Y finalmente propuestas que identifican las asimetrías existentes que obstaculizan una coproducción de conocimiento mutuo en la investigación inter y TD (Schmidt y Neuburger, 2017), así como cuestionamientos fundamentales sobre cómo se conceptualiza el aprendizaje transdisciplinario, y se infiere ampliamente sobre el big data y lo que esto representa en la práctica (Lodge et al., 2017).

Pues bien, de manera general son propuestas — en su mayoría cursos y programasen las que se pretende que los estudiantes apliquen sus habilidades y sus diversos conocimientos en entornos realistas y transdisciplinares para resolver problemas complejos no triviales (Czaplinski et al., 2019) a partir del diseño de metodologías para estructurar cursos, métodos didácticos, sistemas o modelos con enfoque TD. Algunos de los más relevantes para esta investigación son la metodología de los seis pasos que propone Beecroft (2018), que va desde la preparación hasta la terminación de un proyecto TD, en donde los estudiantes trabajan en equipo y se les asignan tareas clave en cada fase. La descripción de métodos didácticos para los cursos que hace Fiala et al. (2018). El sistema de Jia et al. (2019), 


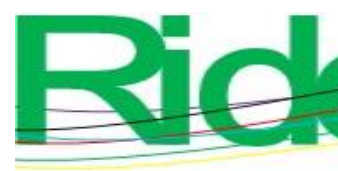

Revista Iberoamericana para la Investigación y el Desarrollo Educativo ISSN 2007 - 7467

que incluye módulos y cursos basados en el enfoque de competencias clave en sostenibilidad, como lo son la competencia interpersonal, el pensamiento sistémico, la competencia estratégica, la competencia anticipatoria y la competencia normativa. Asimismo, el modelo de Acevedo-Osorio et al. (2020), que muestra las fases de los proyectos TD y las implicaciones de actores y ciclos de aprendizaje en espacios inter y TD. Y el modelo de Khoo et al. (2018), que conceptualiza la inter y la TD a partir de convergencias, divergencias y emergencias.

Si bien cada vez son más significativos los progresos TD en la ES (Laasch et al., 2020), aún no son muy visibles las propuestas que detallan las fases para transitar del nivel disciplinario a la TD. Asimismo, no se identifican modelos integrales sistémicos en los que se visualice el cambio de los estudiantes. En cuanto al desarrollo de competencias TD, las propuestas no muestran de manera precisa cómo los estudiantes van evolucionando.

Por lo tanto, el objetivo de esta investigación fue diseñar un proceso transdisciplinario para la ES centrado en el estudiante y en el vínculo IES, industria y sociedad, con base en la teoría holónica, la metodología de los sistemas suaves y la metodología de stage-gates, con el fin de desarrollar el pensamiento sistémico, el trabajo colaborativo y la creatividad en los futuros profesionales, ciudadanos y responsables de la construcción del tejido social en un mundo sostenible.

\section{Metodología}

La presente es una investigación cualitativa (Creswell y Creswell, 2018), de tipo documental, la cual se llevó a cabo en dos fases. En la primera se efectuó una revisión de la frontera del conocimiento en las publicaciones sobre la TD y la educación superior como elementos significativos en la construcción del tejido social. La revisión de la literatura (Jesson, Mathenson y Lacey, 2011; Snyder, 2019) fue de artículos científicos indexados en la Web of Science (WoS), publicados de enero del 2017 a enero de 2021. En ese proceso se determinó cuál era la finalidad del artículo y cuál era la metodología empleada (Jesson, Mathenson y Lacey, 2011). Este trabajo consistió en lo siguiente:

1. Recopilación de la información: Se buscaron artículos científicos indexados en la WoS con el tema transdisciplinary. Posteriormente, se refinó la búsqueda con base en dos criterios: título (transdisciplinary) y tema (high education). Luego se ubicaron y descargaron los artículos para su revisión de manera individual. 


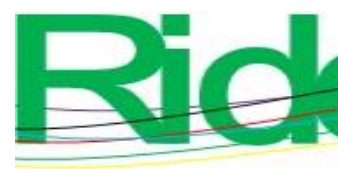

Revista Iberoamericana para la
Investigación y el Desarrollo Educativo
ISSN $2007-7467$

2. Análisis de la información: Se revisaron las publicaciones en las que se identificaron el propósito del artículo, el autor, el país de origen y la metodología empleada.

3. Resultados de la información: Se identificaron coincidencias de los autores e insuficiencias en la investigación sobre TD y educación superior con el fin de diseñar el proceso transdisciplinario para la educación superior (PTD-ES).

En la segunda fase, se diseñó el PTD-ES, el cual se configuró en siete etapas a partir de la revisión teórica:

1. Definición de los momentos del proceso: Disciplinario, multidisciplinario, interdisciplinario y TD a partir de los niveles TD de Nicolescu (1999).

2. Definición de la identidad y complejidad de cada momento del proceso con base en el proceso evolutivo de la teoría holónica de Wilber (1996), en donde cada momento se concibe como un holón que se integra al momento siguiente, generando una nueva identidad.

3. Determinación de las competencias TD por desarrollar en los estudiantes en cada momento del proceso, de acuerdo con la EDS (Unesco, 2020), la propuesta de Muhar, Visser y van Breda (2013) y Wilber (1996).

4. Determinación de compuertas entre cada momento del proceso con base en la metodología stage-gates de Cooper (2008).

5. Determinación de las actividades para la investigación TD para cada momento del proceso a partir de la metodología de los sistemas blandos de Checkland (2000).

6. Definición de los métodos de enseñanza y aprendizaje para el desarrollo de competencias TD con base en el enfoque constructivista de Piaget (1972) y Freire (1983).

7. Integración de los procesos sustantivos de la propuesta: investigación, formación y vinculación con la sociedad.

\section{Resultados}

\section{Proceso transdisciplinario para la educación superior (PTD-ES)}

A partir de la necesidad de formar profesionales y ciudadanos críticos que den solución a los problemas de esta sociedad compleja, es imperante promover ambientes que contribuyan a que los estudiantes desarrollen la capacidad de reflexionar acerca de su rol en el mundo y el significado de lo que estudian y sus objetivos en la vida (Pop, Fotea y Fotea, 

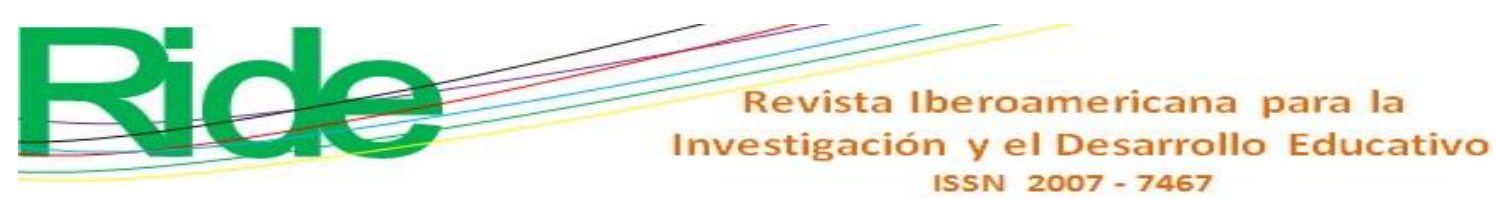

2018; Roysen y Cruz, 2020). Para ello, la clave del desarrollo sostenible y la EDS es promover la participación colectiva (Mokhele y Pinfold, 2020) y la integración de saberes que conduzcan hacia una nueva configuración de pensamiento (Morin, 1996).

En otras palabras, se requieren propuestas centradas en la formación de competencias transdisciplinares de los estudiantes de la ES por medio de la teoría y la práctica (Fiala et al., 2018), donde los agentes participantes asuman el rol de sujetos del proceso que trabajan conjuntamente, de tal manera que la educación sea vista como la problematización de los seres humanos en sus interacciones con el mundo (Freire, 1983).

Atendiendo a estas necesidades, se configura el proceso transdisciplinario para la educación superior (PTD-ES) con el fin de que los estudiantes desarrollen la capacidad de abordar, de manera colectiva, problemas profesionales y sociales. Por eso, es una propuesta centrada en el estudiante, es decir, cimentada en el constructivismo, el enfoque por competencias, así como el enfoque holónico y la metodología de los sistemas suaves, como se observa en la figura 1.

Figura 1. Proceso transdisciplinario para la ES

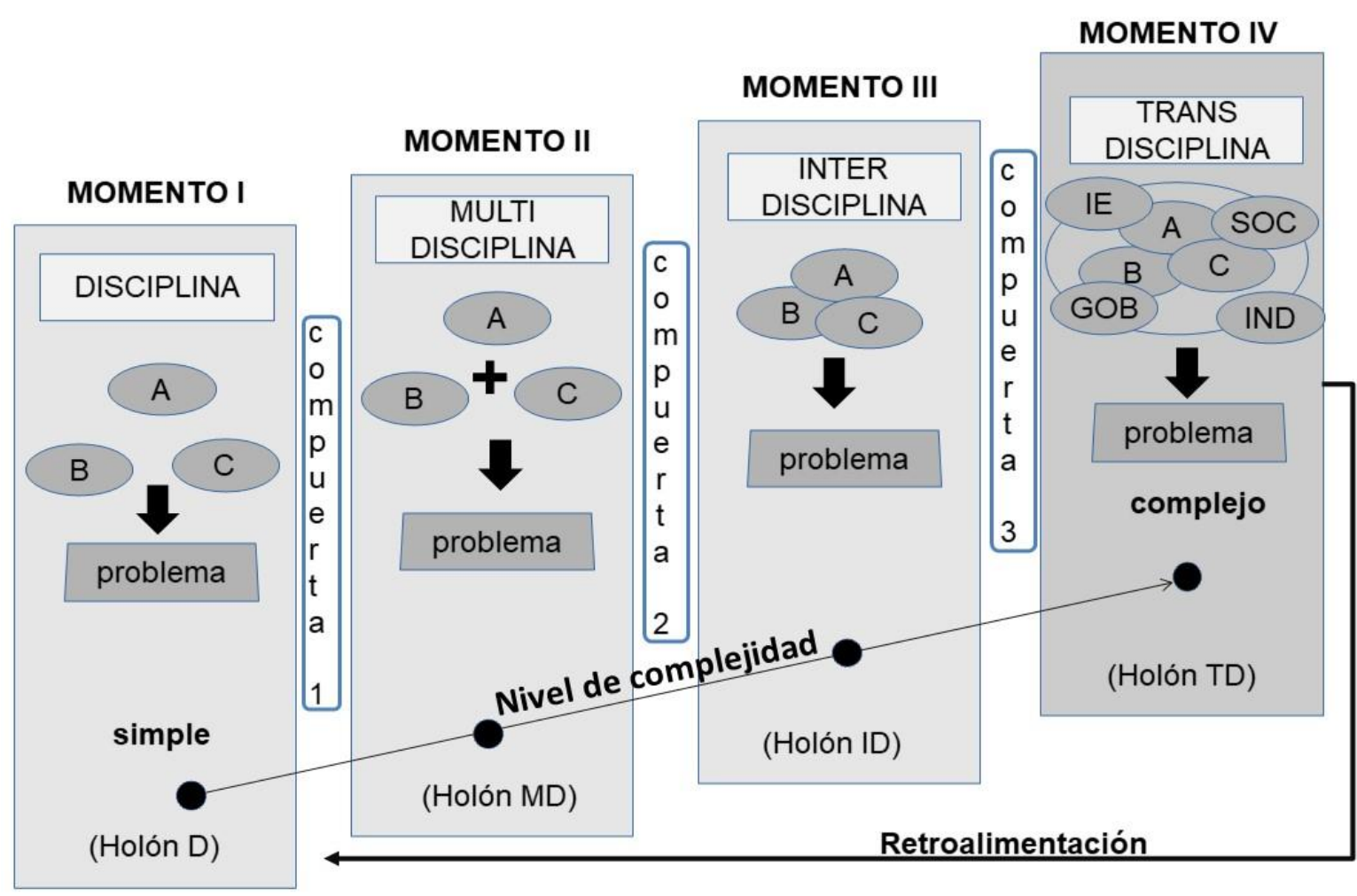

Fuente: Elaboración propia 


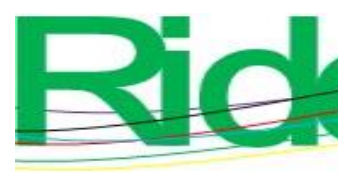

\section{Revista Iberoamericana para la Investigación y el Desarrollo Educativo ISSN 2007 - 7467}

El proceso está compuesto por cuatro momentos: I) disciplina, II) multidisciplina, III) interdisciplina, y, IV) transdisciplina. Cada momento tiene actividades definidas, con base en la metodología de Checkland (2000), que contribuyen a que el estudiante desarrolle un nivel de competencia necesario para transitar al siguiente momento, por lo que cada momento se concibe como un holón en el que se va generando una nueva identidad (Wilber, 1996). Es un proceso cíclico en el que el último momento retroalimenta al primero.

Cabe resaltar que entre cada momento existe una compuerta que se utiliza para verificar que los estudiantes han desarrollado las competencias profesionales esperadas del momento anterior inmediato. A partir de una evaluación realizada, por parte del profesor y de los estudiantes, se determina si cuenta con el nivel de competencias TD necesarias para transitar al siguiente momento o si es necesario reiniciar.

Como se muestra en la figura 1, en el momento I el problema se trata desde cada disciplina, por lo que está representado por las letras A, B y C, las cuales trabajan de manera aislada. En el momento II, las disciplinas, representadas por las mismas letras del momento I, atienden el mismo problema, es decir, se suman para resolverlo desde su disciplina, por eso aparece el símbolo de sumatoria (+). En el momento III, las disciplinas se interrelacionan para atender el mismo problema. Y en el momento IV, las disciplinas se interrelacionan para atender el problema junto con las instituciones de educación superior (IES), la industria (IND), gobierno (GOB) y sociedad (SOC), concibiendo el contexto multidimensional y referencial.

El PTD-ES tiene como eje central el propiciar la investigación TD a través del fortalecimiento del pensamiento sistémico, el trabajo colaborativo y la creatividad, así como del vínculo IES, industria y sociedad, en un espacio de interacción híbrido entre lo académico y la sociedad (Beecroft, 2018). La configuración del vínculo es el medio para atender y entender el contexto, de tal manera que el proceso se desarrolla en la realidad compleja, multidimensional y multirreferencial donde el diálogo TD entre participantes es esencial para resolver problemas tan complejos del mundo actual y para el desarrollo sostenible (Lang et al., 2012; Fiala et al., 2018). Por eso, se consideran como principales métodos didácticos el aprendizaje basado en problemas (Thomas, 2009), el trabajo en equipo (Ingram y Desombre, 1999; Tobón, 2013), la inducción al esquema referencial (Calviño, 2005), la discusión (Freire, 1983) y la alfabetización informacional (Association of College and Research Libraries [ACRL], 2000). 


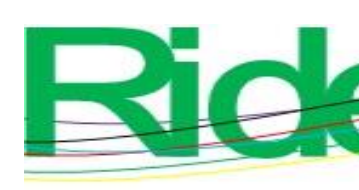

\section{Revista Iberoamericana para la Investigación y el Desarrollo Educativo ISSN 2007 - 7467}

Por tanto, la metodología TD no reemplaza la metodología de cada disciplina, y la sostenibilidad sirve como una imagen integradora. Así bien, los momentos que constituyen el PTD-ES, están basados en los niveles de TD (Nicolescu, 1999), la teoría holónica (Wilber, 1996), la metodología stage-gates (Cooper, 2008) y en la metodología de Checkland (2000) que utiliza una secuencia ordenada de cinco pasos de proceso que bien pueden repetirse en caso de que sea necesario hacer ajustes a pasos anteriores o a la información.

A continuación, se describen los cuatro momentos del PTD-ES con su respectiva compuerta:

Momento I: Disciplina. Se inicia con la preparación del proyecto de investigación TD, por lo que es importante planificar quiénes serán los participantes o "socios de práctica" del proyecto y el lugar donde se llevará a cabo con el fin de facilitar un contacto inicial y familiarizar al equipo de trabajo, ya que es imprescindible que exista la confianza para comprometerse con la cooperación, el intercambio de conocimiento y la comprensión mutua de los respectivos intereses en el proyecto (Baumber et al., 2020; Hernández-Soto, Rodríguez-Medina y Gutiérrez-Ortega, 2020). Asimismo, se desarrolla el prefacio del proyecto de investigación TD, por lo que es necesario introducir a los estudiantes en los aspectos de sostenibilidad relacionados con la disciplina que estudian y la metodología del proyecto, la clarificación reflexiva y mutua de los intereses y objetivos de los estudiantes (Baumber et al., 2020).

Posteriormente, los estudiantes realizan la investigación desde su campo del conocimiento con el fin de fortalecer y/o desarrollar los conocimientos científicos y metodológicos de su disciplina para su posterior participación y aportación al proyecto TD. Cabe señalar que es intencionado que el estudiante entienda, desde un pensamiento simple y un nivel de la realidad, el problema de investigación.

Los métodos de enseñanza y aprendizaje son clave para el desempeño profesional de los estudiantes, ya que son los medios por las cuales desarrollan sus capacidades cognitivas, de comportamiento y afectivas (Cooper, 1999). Los métodos didácticos esenciales en todo momento del PTD-ES son el aprendizaje basado en problemas, con el fin de que los estudiantes aprendan a pensar en lugar de qué pensar dentro del marco de la sostenibilidad (Thomas, 2009). Inducir a la identificación e indagación del esquema referencial, ya que, con base en sus experiencias y conocimientos, los estudiantes piensan, actúan y perciben los fenómenos del mundo (Calviño, 2005). La alfabetización informacional tanto de los profesores como de los estudiantes, pues el acceso, la cantidad y la diversidad de los recursos 


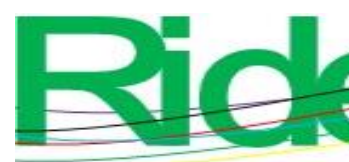

Revista Iberoamericana para la
Investigación y el Desarrollo Educativo
ISSN $2007-7467$

de información no garantizan su calidad y buen uso. Por ello, es indispensable contar con elementos de juicio y habilidades técnicas para hacer este proceso eficiente y efectivo (ACRL, 2000). La discusión considera la acción educativa como una comunicación dialógica con la realidad, con los otros y con la propia conciencia (Freire, 1983). Y el trabajo en equipo, ya que a partir de la configuración de un equipo de trabajo se busca que los estudiantes se comprometan con un objetivo en común y logren un mayor aprendizaje y desarrollo, lo que incluye el trabajo colaborativo, la comunicación, el respeto recíproco, el conocimiento compartido y la resolución de problemas (Ingram y Desombre, 1999; Tobón, 2013). Cabe señalar que son métodos que deben utilizarse en el aula, y es preciso que el profesor se conciba no solo como "el que enseña", sino como el que sigue aprendiendo en un esfuerzo colectivo (Freire, 1983).

Compuerta 1. Con base en el proyecto disciplinario que elaboraron los estudiantes y los resultados obtenidos por los métodos didácticos, se valora que el alumno haya desarrollado seis capacidades esenciales para transitar al momento II, las cuales son: i) conocimientos teóricos disciplinarios necesarios para desarrollar el proyecto TD. ii) Capacidad para reconocer la necesidad de información y la habilidad de localizarla, de tal manera que sea suficiente, vigente, válida y pertinente (ACRL, 2000). iii) Capacidad de comunicación con los integrantes de su equipo disciplinario y con el profesor. iv) Capacidad para conectar la teoría con la investigación y la práctica. v) Capacidad para trabajar con personas de su mismo nivel de estudios y disciplina. vi) Capacidad de abordar y analizar los problemas del mundo desde un pensamiento simple y un solo nivel de la realidad (Choi y Pak, 2006; Nicolescu, 1999). Se espera que al concluir el siguiente momento multidisciplinario, el estudiante mejore el nivel de las competencias desarrolladas en el momento disciplinario.

Momento II: Multidisciplina. Está enfocado en estudiar el problema de la investigación TD desde varias disciplinas de manera simultánea bajo el marco de investigación de cada disciplina (Nicolescu, 1999). Para desarrollar este momento, se requiere la colaboración de todos los participantes del proyecto TD. Se recomienda trabajar de manera conjunta con otras disciplinas, ya sean de la misma IES o de otras.

Debido a que todos provienen de diferentes plataformas, y su manera de pensar y paradigmas son desiguales, es necesario comenzar por definir los conceptos clave y la coincidencia de paradigmas, con la intención de lograr la cooperación y comprensión para el 


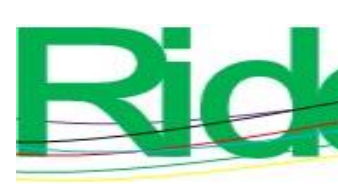

Revista Iberoamericana para la
Investigación y el Desarrollo Educativo
ISSN $2007-7467$

desarrollo de la investigación. Se sugiere articular los términos y conceptos desde una óptica disciplinaria a través de un enfoque Delphi (Checkland, 2000).

A partir de la configuración de los conceptos clave y un objetivo en común, los participantes trabajan, de forma independiente y desde su disciplina, el problema de investigación (Checkland, 2000). Por consiguiente, los estudiantes realizan la investigación con independencia metodológica, conceptual y epistemológica de su disciplina, con ayuda del profesor.

Durante este momento, se recomienda utilizar los métodos de enseñanza y aprendizaje en el aula que se proponen en el primer momento: el aprendizaje basado en problemas, la alfabetización informacional, la discusión en clase y el trabajo en equipo. Es importante señalar que aun cuando son los mismos métodos, la intención es mejorar las competencias de los estudiantes.

Compuerta 2. Al término de la investigación, se hace la valoración del nivel de competencias de los estudiantes, lo cual es necesario para decidir si puede transitar al siguiente momento del proceso o reiniciar el momento. Se identifican cuatro capacidades esenciales: i) dominio de conocimientos disciplinarios clave para la investigación TD, ii) capacidad para buscar, localizar, evaluar y usar información (suficiente, vigente, válida y pertinente), iii) capacidad de comunicación y trabajo con personas de diversas disciplinas, iv) capacidad de abordar, analizar y dar solución a los problemas del mundo, en paralelo con otras disciplinas, desde un pensamiento simple y concibiendo un solo nivel de la realidad (Choi y Pak, 2006; Nicolescu, 1999). Se espera que al concluir el siguiente momento interdisciplinario, el estudiante mejore el nivel de las competencias desarrolladas en el momento multidisciplinario.

Momento III: Interdisciplina. Consiste en transferir métodos de una disciplina a otra. Considerando que interdisciplinariedad tiene tres grados — de aplicación, epistemológico y de generación de nuevas disciplinas (Nicolescu, 1999)—, el PTD-ES se enfoca en el nivel de aplicación sin dejar de ambicionar alcanzar los otros dos, ya que el último grado contribuye al "big bang disciplinario" (Nicolescu, 1999).

Es importante hacer hincapié en que este momento no es para coordinar resultados del momento anterior, sino se concibe como un proceso, por lo que el equipo de investigación sigue siendo multidisciplinario, solo que se utilizará una metodología interdisciplinaria que obligue al estudio de un sistema complejo, lo cual solo se puede lograr con marcos epistémicos, conceptuales y metodológicos compartidos. Así pues, la metodología debe 


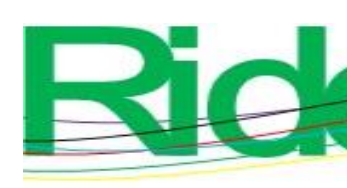

Revista Iberoamericana para la
Investigación y el Desarrollo Educativo
ISSN $2007-7467$

servir para analizar los procesos del sistema, entender su comportamiento y evolución como un todo organizado (García, 2006).

Por consiguiente, la primera actividad es el encuadre y alcance de la investigación TD, para lo cual es necesaria la colaboración de todos los participantes del proyecto TD. Es una actividad en la que previamente se identifica y estructura el problema sin dejar que dominen alguna ideología disciplinaria o valores de los participantes (Checkland, 2000).

Posteriormente, se configura un panorama holístico que identifique al sistema central; para esto, se tiene que llevar a cabo una rigurosa revisión de literatura especifica de la disciplina concerniente al tema a investigar, la cual será perfeccionada por debates de expertos (Checkland, 2000), de tal manera que los participantes, en particular los estudiantes, infieran los atributos y las dimensiones de la investigación desde su disciplina y experiencia individual. Se recomienda utilizar a modo de plataforma para el debate la técnica de la caja morfológica integrada (Zwicky, 1969), en la que la colaboración es clave para definir las características más precisas del sistema y los límites correspondientes (Checkland, 2000). Después de esto, se identifican las actividades más importantes a partir de la descomposición del problema complejo, para lo cual los participantes del proyecto TD tienen que desglosar los procesos, los subprocesos y finalmente las actividades (Checkland, 2000). Por ende, la participación del experto en sistemas es clave para el desarrollo de esta actividad.

Durante este momento también se utilizan los métodos de enseñanza y aprendizaje en el aula, tales como el aprendizaje basado en problemas, la alfabetización informacional, la discusión en clase, el trabajo en equipo y la integración de saberes con el fin de elevar el nivel de las competencias desarrolladas por los estudiantes en el momento multidisciplinario.

Compuerta 3. La valoración del nivel de competencias se realiza, principalmente, con base en la participación que tuvieron los estudiantes para inferir los atributos y dimensiones de la investigación. Se evalúan seis capacidades: i) capacidad de dominar su disciplina e incorporar conocimientos de otras disciplinas (Choi y Pak, 2006; Nicolescu, 1999), ii) capacidad de proveer información para la generación de conocimiento, iii) capacidad de comunicación (configurada desde la relación pensamiento-lenguaje-contexto o realidad), iv) capacidad de trabajar colaborativamente con personas de diferentes campos del conocimiento, v) capacidad de pensar la realidad desde diferentes ópticas bajo un pensamiento abierto y complejo (Nicolescu, 1999; Choi and Pak, 2006), y vi) valorar su participación en la construcción del tejido social. Se espera que al concluir el siguiente 


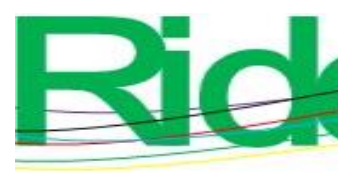

Revista Iberoamericana para la
Investigación y el Desarrollo Educativo
ISSN $2007-7467$

Durante este momento, se pretende que los estudiantes, a partir de su participación en la investigación TD y la aplicación de métodos de enseñanza y aprendizaje, desarrollen, principalmente, seis competencias: la primera, ser capaces de trabajar colaborativamente con personas que tienen diferentes saberes, formas de pensar y ver el mundo. La segunda, ser capaces de examinar los argumentos, integrar y evaluar la información. La tercera, tener la habilidad para sintetizar, producir y generar nuevas ideas. La cuarta, utilizar la discusión para desarrollar un pensamiento crítico, reflexivo y emancipador que permita cuestionarse a sí mismos y al mundo (Jantsch, 1972; Freire, 1983), para lo cual la cultura informacional les permitirá interpretar y hacer juicios informados, además de convertirse en generadores de información (Badwen, 2002). Y la sexta, ser capaces de ver la realidad como un todo y luego en partes (Choi y Pak, 2006; Nicolescu, 1999).

En pocas palabras, a través de la investigación se pretenden construir procesos sociales en los cuales tanto estudiantes como profesores comprendan y atiendan los desafíos y problemas del mundo actual desde el aula y el vínculo con la industria y sociedad, lo cual es fundamental para la generación de conocimiento de manera colectiva para la construcción del tejido social. Finalmente, la TD está enfocada en el conocimiento de uno mismo y en la integración de saberes, lo que deriva en una autotransformación (Nicolescu, 1999) con la intención de configurar una sociedad sostenible.

\section{Discusión}

Existen modelos y metodologías para comprender y conceptualizar la inter y TD, así como propuestas de programas y cursos TD para la educación superior, como la metodología de Beecroft (2018) que identifica seis fases para el proyecto TD, los métodos didácticos para los cursos TD que proponen Fiala et al. (2018), el sistema de Jia et al. (2019) basado en el enfoque de competencias clave en sostenibilidad, el modelo de Acevedo-Osorio et al. (2020) que propone fases de los proyectos TD e implicaciones de actores y ciclos de aprendizaje en espacios inter y TD, el modelo de Khoo et al. (2018) que determina convergencias, divergencias y emergencias de la inter y la TD, entre otros.

En este contexto, el PTD-ES se construye desde los niveles de TD (Nicolescu, 1999), la teoría holónica (Wilber, 1996), la metodología stage-gates (Cooper, 2008) y la metodología de Checkland (2000). Es decir, se integran métodos de enseñanza y aprendizaje, así como competencias TD que deben desarrollar los estudiantes de la ES en cada momento 


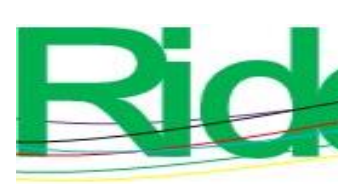

Revista Iberoamericana para la Investigación y el Desarrollo Educativo ISSN $2007-7467$

del proceso. El PTD-ES está centrado en el estudiante sin dejar de lado al profesor, ya que se espera que ambos aprendan en el proceso. Además, es de carácter vinculante, capaz de perfeccionarse con su práctica, objetivo, configuración de las IES y con la organización de los participantes.

Finalmente, cabe señalar que entre las limitaciones del PTD-ES sobresalen que aún no se ha puesto en marcha, por lo que está pendiente su validación. Es un proceso que no atiende las barreras que afectan la eficacia y la eficiencia de la TD, como el dominio del pensamiento disciplinar en la ES, la cultura individualista y egoísta de los profesores, la estructura organizacional de las IES y su falta de interés y compromiso hacia la TD, así como la desvinculación con otras instituciones, la industria y la sociedad, entre otras.

\section{Conclusiones}

El objetivo planteado para esta investigación se cumplió satisfactoriamente. Por ello, se propone el diseño de un proceso cimentado en la transdisciplinariedad, centrado en los estudiantes y en el vínculo IES-industria-sociedad, el cual está dividido en cuatro momentos, que van de lo simple a lo complejo, dado por la concepción de la realidad con la que se aborda, analiza y se da respuesta a los problemas del mundo.

Cada momento tiene actividades específicas para que el estudiante participe y desarrolle competencias TD; principalmente, de pensamiento sistémico, trabajo colaborativo y creatividad, que le permitan comprender y atender los problemas complejos del mundo. Por ende, se propone que el profesor guíe y acompañe en todo momento al estudiante.

La investigación es la base para la enseñanza y el aprendizaje, la cual puede ser reforzada con los métodos didácticos del aprendizaje basado en problemas, la discusión, la alfabetización informacional, la identificación e indagación del esquema referencial y el trabajo en equipo. En cada momento del proceso, el estudiante desarrolla un nivel de competencia, lo que le da una nueva identidad, necesaria para transitar al siguiente momento hasta llegar al TD.

Finalmente, derivado de la revisión de la literatura y los resultados de esta investigación, se sugiere considerar a la acción educativa en las IES como algo dinámico vinculada con la investigación, la realidad y con "todos" lo que son partícipes en la construcción de la sociedad. En tal sentido, resulta fundamental la formación TD de los estudiantes de la ES para su desarrollo personal, su integración al mundo laboral, su 


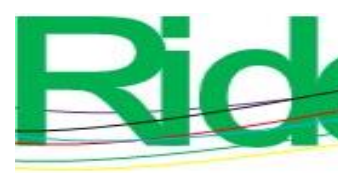

Revista Iberoamericana para la
Investigación y el Desarrollo Educativo
ISSN $2007-7467$

contribución al crecimiento económico del país y, principalmente, su participación en la configuración de un mundo sostenible.

\section{Futuras líneas de investigación}

Se propone como futuras investigaciones llevar a la práctica el PTD-ES, mejorar la propuesta del proceso desde un pensamiento sistémico más integral, así como diseñar un instrumento de medición de competencias TD. No obstante, este proceso tiene implicaciones. De manera general, se visualiza la inversión en bases de datos de alto impacto, desarrollar la cultura informacional, formar a los profesores bajo el enfoque TD, adaptar la estructura organizacional de las IES y fomentar la investigación.

\section{Agradecimientos}

Agradecemos las facilidades otorgadas para la realización de este trabajo al Instituto Politécnico Nacional, a la Secretaría de Investigación y Posgrado (con el proyecto SIP 20210297), a la Unidad Profesional Interdisciplinaria de Ingeniería y Ciencias Sociales y Administrativas (UPIICSA), a la Sección de Estudios de Posgrado e Investigación, al Departamento de Estudios Profesionales Genéricos, al Programa de Estímulo al Desempeño Docente (PEDD del IPN) y al doctorado en Educación de la Universidad OMI Centro de Investigación.

\section{Referencias}

Acevedo-Osorio, Á., Hofmann-Souki, S. and Cruz Morales, J. C. (2020). Holistic competence orientation in sustainability-related study programmes: lessons from implementing transdisciplinary student team research in Colombia, China, Mexico and Nicaragua. Sustainability Science, 15, 233-246. Doi: https://doi:10.1007/s11625019-00687-8.

Association of College and Research Libraries [ACRL]. (2000). Information literacy competency standars for higher education. Chicago: American Library Association. Retrieved from https://hdl.handle.net/10150/105645.

Badwen, D. (2002). Revisión de los conceptos de alfabetización informacional y alfabetización digital. Anales de Documentación, 5, 361-408. 


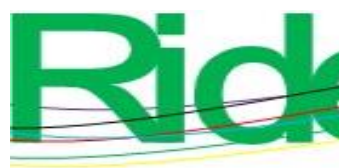

Revista Iberoamericana para la
Investigación y el Desarrollo Educativo
ISSN $2007-7467$

Baptista, B. V. and Rojas-Castro, S. (2020). Transdisciplinary institutionalization in higher education: a two-level analysis. Studies in Higher Education, 45(6), 1075-1092. Doi: https://doi:10.1080/03075079.2019.1593347.

Barakhsanova, E. A., Prokopyev, M. S., Olesova, S. G., Olesov, N. P., Lukina, T. N., Sorochinskiy, M. A. and Tatarinov, F. F. (2020). Transdisciplinary approach to the learning process organization in the e-learning information environment of a college. International Transaction Journal of Engineering, Management, \& Applied Sciences \& Technologies, 11(3), 11A03M. Doi: https://doi:10.14456/ITJEMAST.2020.53.

Bárcena, F. y Mélich, J.-C. (2014). La eduación como acontecimiento ético: natalidad, narración y hospitalidad. Bueno Aires: Miño y Dávila.

Baumber, A., Kligyte, G., van der Bijl-Brouwer, M. and Pratt, S. (2020). Learning together: a transdisciplinary approach to student-staff partnerships in higher education. Higher Education Research \& Development, 39(3), 395-410. Doi: https://doi:10.1080/07294360.2019.1684454.

Beecroft, R. (2018). Embedding Higher Education into a Real-World Lab: A ProcessOriented Analysis of Six Transdisciplinary Project Courses. Sustainability, 10(3798). Doi: https://doi:10.3390/su10103798.

Biberhofer, P. and Rammel, C. (2017). Transdisciplinary learning and teaching as answers to urban sustainability challenges. International Journal of Sustainability in Higher Education, 18(1), 63-83. Doi: https://doi:10.1108/IJSHE-04-2015-0078.

Calviño, M. (2005). Orientación psicológica. Esquema referencial de alternativa múltiple (2. ${ }^{a}$ ed.). La Habana: Editorial Félix Varela.

Century, J., Ferris, K. A. and Zuo, H. (2020). Finding time for computer science in the elementary school day: a quasi-experimental study of a transdisciplinary problembased learning approach. International Journal of STEM Education, 7(20). Doi: https://doi:10.1186/s40594-020-00218-3.

Checkland, P. (2000). Soft Systems Methodology; A 30-year Restrospective. Systems Reserch and Behavioral Science, 17.

Choi, B. and Pak, A. (2006). Multidisciplinarity, interdisciplinarity and transdisciplinarity in health research, services, education and policy: 1. Definitions, objectives, and evidence of effectiveness. Clin Invest Med, 29(6), 351-364. Doi: https://doi.org/10.25011/cim.v30i6.2950. 


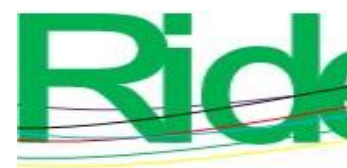

Revista Iberoamericana para la
Investigación y el Desarrollo Educativo
ISSN $2007-7467$

Contreras, P., Jiménez, J., Browne, R. and Oliva-Figueroa, I. (2020). Interfaces university society in the transdisciplinary prospective: Social Communication and Institutionalweb Discourse associated with the organization of knowledge in Universities in Chile. ALPHA(50), 195-208. Doi: https://doi:10.32735/S0718-2201202000050791.

Cooper, J. (1999). Estrategias de enseñanza. Guía para una mejor enseñanza. México: Limusa Noriega Editors.

Cooper, R. (2008). Perspective: The Stage-Gates Idea-to-Launch Process-Update, What's New, and NexGen Systems. The Journal of Product Innovation Management, 25, 213-232. Doi: https://doi: 10.1111/j.1540-5885.2008.00296.x.

Creswell, J. and Creswell, J. (2018). Research Design: Qualitative, Quantitative, and Mixed Methodos Approaches ( $5^{\text {th }}$ ed.). California: SAGE Edge.

Czaplinski, I., Turner, I. W., Helmstedt, K., Corry, P. and Mallet, D. G. (2019). Industrybased, transdisciplinary, complex problems as realistic settings for applying the $M$ in STEM. International Journal of Mathematical Education in Science and Technology. Doi: https://doi:10.1080/0020739X.2019.1692932.

Echegoyen-Sanz, Y. and Martin Ezpeleta, A. (2019). Travelling with darwin and humboldt. a transdisciplinary educational experience. Journal of Education Culture and Society, 10(2), 111-125. Doi: https://doi:10.15503/jecs20192.111.125.

Fiala, V., Freyer, B., Klimek, M. and Fahringer, A. (2018). How do you teach transdisciplinary competences for food and farming systems research? Insights from the course System Analysis and Scenario Technique. Open Agriculture, 3, 553-566. Doi: https://doi:10.1515/opag-2018-0059.

Freire, P. (1983). Pedagogía del oprimido. Ciudad de México: Siglo Veintiuno Editores.

García, R. (2006). Sistemas complejos. Madrid: Editorial Gedisa.

Garutsa, T. C. and Mahlangu, P. M. (2019). A Transdisciplinary Approach and Indigenous Knowledge as Transformative Tools in Pedagogical Design: The Case of the Centre for Transdisciplinary Studies, University of Fort Hare. Africa Education Review, 16(5), 60-69. Doi: https://doi:10.1080/18146627.2016.1251293.

Giebels, D., Caurus, J., Paul, M., Kleyer, M., Siebenhüner, B., Arns, A., Bartholoma, A., Carlow, V., Jürgen, J., Tietjen, B., Wehrmann, A. and Schroder, B. (2020). Transdisciplinary knowledge management: A key but underdeveloped skill in EBM $\begin{array}{llll}\text { decision-making. } & \text { Marine } & \text { Policy, }\end{array}$ https://doi.org/10.1016/j.marpol.2020.104020. 


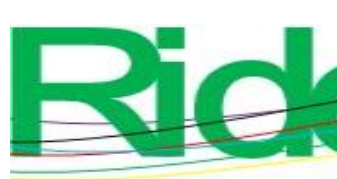

Revista Iberoamericana para la Investigación y el Desarrollo Educativo ISSN $2007-7467$

Hernández-Soto, R., Rodríguez-Medina, J. and Gutiérrez-Ortega, M. (2020). Trust and knowledge sharing in a transdisciplinary community of practice: a convergent parallel case study. Revista Latinoamericana de Tecnología Educativa, 19(2), 47-63. Doi: https://doi:10.17398/1695-288X.19.2.47.

Holhl, S., Knerr, S. and Thompson, B. (2019). A framework for coordination center responsibilities and performance in a multi-site, transdisciplinary public health research initiative. Research Evaluation, 28(3), 279-289. Doi: https://doi:10.1093/reseval/rvz012.

Ingram, H. and Desombre, T. (1999). Teamwork in health care: lessons from the literature and from good practice around the world. Journal of Management in Medicine, 13, $51-8$.

Jackson, M. (1994). Más allá de las modas administrativas: el pensamiento sistémico para los administradores. Innovar, 4, 6-21.

Jaeger, W. (2001). Paideia: Los ideales de la cultura griega. Ciudad de México: Fondo de Cultura Económica.

Jantsch, E. (1972). Inter- and transdisciplinary university: a systems approach to education and innovation. Higher Education, 1(1), 7-37. Doi: https://www.jstor.org/stable/3445957.

Jesson, J. K., Mathenson, L. and Lacey , F. M. (2011). Doing Your Literature Review: Traditioanl and Systematic Techniques. London: SAGE Publications.

Jia, Q., Wang, Y. and Fengting, L. (2019). Establishing transdisciplinary minor programme as a way to embed sustainable development into higher education system Case by Tongji University, China. International Journal of Sustainability in Higher Education, 20(1), 157-169. Doi: https://doi:10.1108/IJSHE-05-2018-0095.

Kant, I. (2003). La crítica de la razón pura. Bueno Aires: Losada.

Keller, L., Stoetter, J., Oberrauch, A. and Kuthe, A. (2019). Changing Climate Change Education Exploring moderate constructivist and transdisciplinary approaches through the research-education co-operation k.i.d.Z.21. GAIA, 28(1), 35-43. Doi: https://doi:10.14512/gaia.28.1.10.

Khoo, S.-M., Haapakoski, J., Hellstén, M. and Malone, J. (2018). Moving from interdisciplinary research to transdisciplinary educational ethics: Bridging epistemological differences in researching higher education internationalization(s). 


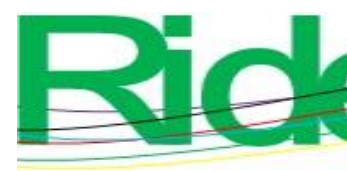

Revista Iberoamericana para la
Investigación y el Desarrollo Educativo
ISSN $2007-7467$

Muhar, A., Visser, J. and van Breda, J. (2013). Experiences from establishing structured inter- and transdisciplinary doctoral programs in sustainability: a comparison of two cases in South Africa and Austria. Journal of Cleaner Production, 61, 12-129. Doi: https://doi:10.1016/j.jclepro.2013.07.031.

Nicolescu, B. (1999). La evolución transdisciplinaria del aprendizaje. Trans-pasando Fronteras, 4, 39-50. Doi: https://doi:10.18046/retf.i4.1779.

Norden, B. (2018). Transdisciplinary teaching for sustainable development in a whole school project. Environmental Education Research, 24(5), 663-677. Doi: https://doi:10.1080/13504622.2016.1266302.

Oliva-Figueroa, I. G., Molina-Chavez, W. M., Quintero-Tapia, J. J. and Diaz-Barrera, N. S. (2018). Transdisciplinary prospective and knowledge organization: an epistemyc approach to higher education in the magallanes region, Chile. MAGALLANIA, 46(2), 129-142. Doi: https://doi:10.4067/S0718-22442018000200129.

Organización de las Naciones Unidas para la Educación, la Ciencia y la Cultura [Unesco]. (2014). Shaping the future we want. UN decade of education for sustainable development (2005-2014). París: UNESCO.Re

Organización de las Naciones Unidas para la Educación, la Ciencia y la Cultura [Unesco]. (2020). Educación para el Desarrollo Sostenible. Hoja de ruta. París: UNESCO.

Piaget, J. (1972). Intellectual Evolution from Adolescence to Adulthood. Human Development, 15, 1-12. Doi: https://doi: 10.1159/000112531.

Pop, I. G., Fotea, I. S. and Fotea, S. L. (2018). Innovation networking, knowledge transdisciplinary spaces. Studia Universitatis Vasile Goldis Arad Seria Stiinte Economice, 28(2), 86-108. Doi: https://doi:10.2478/sues-2018-0011.

Rammel, C., Velázquez, L. and Mader, C. (2015). Sustainability assessment in higher education institutions: what and how? En M. Barth, G. Michelsen, M. Rieckmann and I. Thomas, Routledge Handbook of Higher Education for Sustainable Development (pp. 112-131). London: Routledge.

Reme, S., Caban-Martinez, A., Young, J., Arlinghaus, A. and Gray, G. (2015). A model for development and delivey of a graduate course in transdisciplinary research. Public Health Reports, 130. Doi: https://doi.org/10.1177/003335491513000520.

Roysen, R. and Cruz, T. C. (2020). Educating for transitions: ecovillages as transdisciplinary sustainability "classrooms". International Journal of Sustainability in Higher Education, 21(5), 977-992. Doi: https://doi:10.1108/IJSHE-01-2020-0009. 


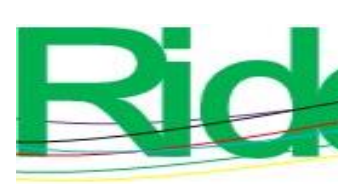

Revista Iberoamericana para la
Investigación y el Desarrollo Educativo
ISSN $2007-7467$

Rupnik, D. and Avsec, S. (2020). Effects of a transdisciplinary educational approach on students' technological literacy. Journal of Baltic Science Education, 19(1), 121-141. Doi: https://doi:10.33225/jbse/20.19.121.

Salgado, R. (2015). Encuentro, voces y evoca-acciones. Anudamientos metodológicos para el estudio del vinculo jóvenes, escuela secundaria y comunidad en tiempos de incertidumbre. Uaricha. Revisita de Psicología, 12(27), 45-56.

Schmidt, L. and Neuburger, M. (2017). Trapped between privileges and precariousness: Tracing transdisciplinary research in a postcolonial setting. Futures, 93, 54-67. Doi: https://doi:10.1016/j.futures.2017.07.005.

Scott, C. (2015). El futuro del aprendizaje 2. ¿Qué tipo de aprendizaje se necesita en el siglo XXI? Investigación y Prospectiva en Educación. Documentos de trabajo, 1-19. Recuperado de http://repositorio.minedu.gob.pe/handle/123456789/4661.

Serviss, T. and Voss, J. (2019). Researching Writing Program Administration Expertise in Action:A Case Study of Collaborative Problem Solving as Transdisciplinary Practice. College Composition and Communication, 70(3), 446-475.

Sipos, Y., Battisti, B. and Grimm, K. (2008). Achieving transformative sustainability learning: engaging head, hands and heart. International Journal of Sustainability in Higher Education, 9(1), 68-86. Doi: https://doi:10.1108/14676370810842193.

Snyder, H. (2019). Literature review as a research methodology: An overview and guidelines. Journal of Business Research, 104, 333-339. Doi: https://doi:10.1016/j.jbusres.2019.07.039.

Tasdemir, C. and Gazo, R. (2020). Integrating sustainability into higher education curriculum through a transdisciplinary perspective. Journal of Cleaner Production, 265, 121759. Doi: https://doi:10.1016/j.jclepro.2020.121759.

Thomas, I. (2009). Critical thinking, transformative learning, sustainable education, and Problem-Based Learning in Universities. Journal of Transformative Education, 7, 245-264. Doi: https://doi:10.1177/1541344610385753.

Tobón, S. (2013). Formación integral y competencias. Pensamiento complejo, currículo, didáctica y evaluación. Bogotá: ECOE.

Wilber, K. (1996). Breve historia de todas las cosas. Barcelona: Editorial Kairós . Zwicky, F. (1969). Discovery, Invention, Research - Through the Morphological Approach. New York: Macmillan. 


\begin{tabular}{|c|c|}
\hline Rol de Contribución & Autor (es) \\
\hline Conceptualización & Graciela Salgado-Escobar «principal» \\
\hline Metodología & Graciela Salgado-Escobar «principal» \\
\hline Software & No aplica. \\
\hline Validación & $\begin{array}{l}\text { Graciela Salgado-Escobar «igual» Mario Aguilar-Fernández } \\
\text { «igual» }\end{array}$ \\
\hline Análisis Formal & $\begin{array}{l}\text { Graciela Salgado-Escobar «igual» Mario Aguilar-Fernández } \\
\text { «igual» }\end{array}$ \\
\hline Investigación & $\begin{array}{l}\text { Graciela Salgado-Escobar «principal» Mario Aguilar- } \\
\text { Fernández «que apoya» }\end{array}$ \\
\hline Recursos & $\begin{array}{l}\text { Graciela Salgado-Escobar «igual» Mario Aguilar-Fernández } \\
\text { «igual» }\end{array}$ \\
\hline Curación de datos & $\begin{array}{l}\text { Graciela Salgado-Escobar «igual» Mario Aguilar-Fernández } \\
\text { «igual» }\end{array}$ \\
\hline $\begin{array}{l}\text { Escritura - Preparación del } \\
\text { borrador original }\end{array}$ & $\begin{array}{l}\text { Graciela Salgado-Escobar «principal» Mario Aguilar- } \\
\text { Fernández «que apoya» }\end{array}$ \\
\hline $\begin{array}{l}\text { Escritura - Revisión y } \\
\text { edición }\end{array}$ & $\begin{array}{l}\text { Graciela Salgado-Escobar «principal» Mario Aguilar- } \\
\text { Fernández «que apoya» }\end{array}$ \\
\hline Visualización & $\begin{array}{l}\text { Graciela Salgado-Escobar «principal» Mario Aguilar- } \\
\text { Fernández «que apoya» }\end{array}$ \\
\hline Supervisión & $\begin{array}{l}\text { Graciela Salgado-Escobar «principal» Mario Aguilar- } \\
\text { Fernández «que apoya» }\end{array}$ \\
\hline Administración de Proyectos & $\begin{array}{l}\text { Graciela Salgado-Escobar «principal» Mario Aguilar- } \\
\text { Fernández «que apoya» }\end{array}$ \\
\hline Adquisición de fondos & $\begin{array}{l}\text { Graciela Salgado-Escobar «igual» Mario Aguilar-Fernández } \\
\text { «igual» }\end{array}$ \\
\hline
\end{tabular}

